\title{
Reformismo pedagógico, utilitarismo y nacionalismo en las escuelas primarias de la Argentina durante la crisis de 1930
}

\author{
Adrián Ascolani
}

\begin{abstract}
Resumen:
La crisis económica internacional tuvo en la Argentina su mayor impacto en 1932. Sus efectos permanecieron presentes durante más de un decenio. La crisis fiscal afectó el financiamiento de la educación pública, que era predominante en la instrucción primaria. Esta coyuntura compleja tuvo efectos transformadores en cuanto a la organización escolar, produciéndose una convivencia de propuestas y prácticas educativas heterogéneas. El Consejo Nacional de Educación, organismo colegiado compuesto por intelectuales y funcionarios de diferente extracción política, elaboró una reforma moderada del currículo y de las prácticas educativas, que combinaba en forma ecléctica la filosofía espiritualista, la psicología experimental, la renovación de escuela activa y la propaganda patriótica. La orientación de esta transformación educativa fue nacionalista y utilitaria, buscando resolver problemas vitales cotidianos al mismo tiempo que fortalecía la adaptación e integración social. Algunos de sus instrumentos pedagógicos y didácticos se consolidaron por su eficacia y permanecieron vigentes durante décadas. En este artículo se analizan la naturaleza y los propósitos de esta reforma así como las prácticas que buscó instalar. El abordaje metodológico se encuadra en la historia cultural, la microhistoria y la historia prosopográfica.
\end{abstract}

Palabras clave:

espiritualismo pedagógico; educación nacionalista; reforma educativa; prácticas educativas 


\title{
Pedagogical reformism, utilitarianism and nationalism in the primary schools of Argentina during the crisis of 1930.
}

\begin{abstract}
The international economic crisis had its greatest impact in Argentina in 1932. Its effects remained present for more than a decade. The fiscal crisis affected the financing of public education, which was predominant in primary education. This complex conjuncture had transformative effects in terms of school organization, resulting in a coexistence of heterogeneous educational proposals and practices. The National Council of Education, a collegiate body composed of intellectuals and officials of different political extraction, developed a moderate reform of the curriculum and educational practices, which combined in an eclectic way the spiritualist philosophy, experimental psychology, the renovation of active school and the patriotic propaganda. The orientation of this educational transformation was nationalistic and utilitarian, seeking to solve daily life problems at the same time as strengthening social adaptation and integration. Some of its pedagogical and didactic instruments were consolidated for their effectiveness and remained in force for decades. This article analyzes the nature and purposes of this reform as well as the practices it sought to install. The methodological approach is framed in cultural history, microhistory and prosopographic history.
\end{abstract}

Key words: pedagogical spiritualism; nationalist education; education reform; educational practices.

\section{Réformisme pédagogique, utilitarisme et nationalisme dans les écoles primaires d'Argentine pendant la crise de 1930}

Résumé: La crise économique internationale a eu son plus grand impact en Argentine en 1932. Ses effets sont restés présents pendant plus d'une décennie. La crise fiscale a affecté le financement de l'éducation publique, qui prédominait dans l'enseignement primaire. Cette conjoncture complexe a eu des effets de transformation en termes d'organisation scolaire, entraînant la coexistence de propositions et de pratiques éducatives hétérogènes. Le Conseil National de l'Éducation, une instance collégiale composée d'intellectuels et de responsables de différentes appartenances politiques, a mis au point une réforme modérée du curriculum et des pratiques pédagogiques, associant de manière éclectique la philosophie spiritualiste, la psychologie expérimentale, la rénovation de l'école active et la propagande patriotique. L'orientation de cette transformation éducative était nationaliste et utilitaire, cherchant à résoudre les problèmes de la vie quotidienne tout en renforçant l'adaptation et l'intégration sociales. Certains de ses instruments pédagogiques et didactiques ont été consolidés pour leur efficacité et sont restés en vigueur pendant des décennies. Cet article analyse la nature et les objectifs de cette réforme, ainsi que les pratiques qu'elle cherche à mettre en place. L'approche méthodologique est encadrée dans l'histoire culturelle, la microhistoire et l'histoire prosopographique.

Mots clés: spiritualisme pédagogique; éducation nationaliste; réforme de l'éducation; pratiques pédagogiques.

\section{Reformismo pedagógico, utilitarismo e nacionalismo nas escolas primárias da Argentina durante a crise de 1930}

Resumo: A crise económica internacional teve o seu maior impacto na Argentina em 1932. Os seus efeitos perduraram por mais de uma década. A crise fiscal afetou o financiamento da educação pública, que predominou na educação primária. Esta conjuntura complexa teve efeitos de transformação em termos de organização escolar, originando a coexistência de práticas educativas heterogéneas. O Conselho Nacional de Educação, um órgão colegial composto por intelectuais e funcionários de diferentes filiações políticas, desenvolveu uma reforma moderada do currículo e das práticas de ensino, associando de maneira eclética a filosofia espiritualista, a psicologia experimental, a renovação da escola ativa e a propaganda patriótica. A orientação desta transformação educativa era nacionalista e utilitária, tentando resolver os problemas da vida cotidiana, reforçando, ao mesmo tempo, a integração e a adaptação sociais. Alguns dos seus instrumentos pedagógicos e didáticos foram consolidados pela sua eficiência e permaneceram em vigor durante décadas. Este artigo analisa a natureza e os objectivos desta reforma, bem como as práticas que pretende implementar. A abordagem metodológica enquadra-se na história cultural, na microhistória e na história prosopográfica

Palavras-chave: espiritualismo pedagógico; educação nacionalista; reforma da educação; práticas pedagógicas. 


\section{Introducción}

La década de 1930 ha sido estigmatizada hasta no hace muchos años como la "década infame" debido a determinados actos de fraude político y negociados económicos. Fue una década ensombrecida por la crisis económica y la proscripción a fuerzas políticas populares como el sector de la Unión Cívica Radical liderado por el presidente depuesto, Hipólito Yrigoyen, y el Partido Comunista de la Argentina. En la historiografía de la educación han tenido una fuerte influencia estas líneas críticas, de modo que han prevalecido en los estudios desarrollados en los últimos treinta años los abordajes tendientes estudiar el autoritarismo político y las tendencias filo-fascistas (Bejar, 1992), la propaganda nacionalista y el conservadurismo de la Iglesia Católica (Escudé, 1990) en la conducción del sistema educativo. Con la mirada enfocada en la política nacional o en determinados casos provinciales, no se ha valorado la potencialidad de las experiencias político-educativas provinciales, como lo demuestra la relativamente escasa producción de los dos últimos decenios (Ossanna, 1993), y tampoco se han revisado las versiones globales (Puiggrós, 1992) canonizadas a comienzos de la década de 1990, sobre las políticas del Ministerio de Justicia e Instrucción Pública de la Nación y de su dependencia encargada de la educación primaria en su jurisdicción, el Consejo Nacional de Educación (CNE). Algunas obras que abordan el período y están focalizadas en objetos particulares, como la infancia, el currículo escolar, y las asociaciones docentes (Carli, 2006; Gvirtz, 1999; Ascolani y Gindin, 2018) o el propio CNE (Ascolani, 2015) entre otras, permiten apreciar un trasfondo complejo detrás de las escenas y discursos predominantes.

En este artículo nos proponemos mostrar la convivencia ecléctica de decisiones, posicionamientos, y referentes de heterogénea inspiración en el CNE, traducidas en el funcionamiento institucional y en la orientación de la enseñanza. Conservadurismo y progresismo coexistieron y se imbricaron, reflejando las trayectorias individuales heterogéneas de los miembros en este órgano colegiado, a través de los acuerdos y de las resoluciones pragmáticas tomadas, así como por la inercia de los funcionarios de carrera que ocupaban los roles de inspección y tenían firmes posiciones ideológicas y pedagógicas. Hemos analizado los dos años iniciales del gobierno del general Agustín P. Justo, presidente de la Nación desde 1932 hasta 1938: el primero de transición y el segundo de inicio de una reforma educativa que continuaría vigente durante el resto de la década (Ascolani, 2015; 2017, p. 51-55).

\section{Una fase de transición con continuidades}

El CNE era un organismo que se componía de un gobierno colegiado del cual emanaban prácticamente todas las resoluciones sobre el funcionamiento de su estructura 
escolar y administrativa, encuadradas en lo dispuesto por la Ley $N^{\circ} 1420$, de educación común. La Inspección General de Escuelas las hacía ejecutar a través de sus inspectores generales en las tres jurisdicciones que tenía a cargo: Capital Federal, Provincias y Territorios Nacionales -gobernaciones a cargo del Poder Ejecutivo Nacional-. En las provincias, desde 1905, por la Ley $N^{\circ} 4874$, el CNE podía establecer escuelas primarias rurales, si sus gobiernos lo solicitaban, resultando de ello que en la década de 1930 eran más las escuelas rurales nacionales que las provinciales. En abril de 1932, el presidente de la Nación designó al abogado y político Ramón Cárcano en el cargo de presidente del CNE. Figura política y académica de gran trayectoria, era un referente consolidado de la vertiente más liberal de las fuerzas conservadoras opuestas al Partido Radical. ${ }^{1}$ Integraron ese Consejo: el abogado radical Agustín Araya, nombrado vicepresidente, el doctor Agustín Pestalardo y los profesores Avelino Herrera e Ines Recalt como vocales (MEC, junio 1932, sección oficial, p. 1). Su gestión de fue de sólo siete meses, dado que en 1933 Cárcano pasó a ocupar el cargo de embajador en la República del Brasil. El organismo mantuvo el discurso educativo espiritualista instalado en 1930, que concebía a "la superación espiritual de la infancia" como el fin principal de la educación, y la ejemplaridad moral y el honor del educador como los elementos que debía sustentar la vocación, la capacidad técnica y la dignidad profesional. La dicotomía entre educación e instrucción se reavivaba en la medida que las nuevas autoridades concebían a la moral, el "alma", y el amor como elementos imprescindibles, responsabilizando al docente del apostolado que le tocaba realizar, enfatizando la función socializadora de la escuela, en el marco de la educación integral (CNE, 1932, pp. 45-46).

EI CNE difundió la exaltación del sentimiento nacional como objetivo principal de la escuela, con lo cual se reforzaron los rituales, la reverencia a los símbolos patrios y la conmemoración de fechas relacionadas con la independencia, aplicando instrumentos que facilitaran la emotividad infantil, como la música y la literatura (Escudé, 1990). En 1932 el acto patriótico de mayor relieve se desarrolló en la Capital Federal, el día 3 septiembre, honrando al prócer liberal Bernardino Rivadavia. Participaron todas las escuelas de la ciudad y se imprimieron 50.000 volantes que contenían una leyenda que parangonaba a Rivadavia con Jesús. Los desfiles y actos públicos movilizaron a 34.641 alumnos, con una gran concentración de 10.174 escolares (CNE, 1932, p. 50; MEC, septiembre 1932, pp. 5-8). En el discurso nacionalista de la cúpula educativa ya había ganado algún espacio la noción patriótica pacifista relacionada con el trabajo, el honor, la responsabilidad cívica, y eso dio lugar a otras conmemoraciones con fines moralistas, como el día del Día del Arbol, el Día del Animal, y los festejos de fin de curso.

La ideología nacionalista contribuyó a que emergieran las ideas de centralización institucional, en tensión con el federalismo establecido por en la Constitución Nacional 
(Ascolani, 2015, p. 857). Cada provincia y el gobierno federal tenían sus propias leyes educativas, y la evolución institucional bastante autónoma había generado particularidades en la organización escolar. El CNE inició una política de armonización los planes y programas con las provincias, sin alterar los propios, buscando coordinar los estudios de los cuatro grados que ofrecían sus escuelas nacionales rurales -el mínimum fijado por la Ley № 1420 - con el ciclo primario completo que tenían la mayoría de las provincias, que en general era de seis años. En octubre, resolvió gestionar acuerdos con las provincias en torno a acciones conjuntas: a) creación de escuelas de concentración, internados, escuelas ambulantes e inscripción periódica; b) creación los grados $5^{\circ}$ y $6^{\circ}$ a cargo de las provincias utilizando los locales de escuelas nacionales ubicadas en centros urbanos, articulando programas, métodos y procedimientos. No transigiría con en el pedido que las provincias hacían de que pasaran a su jurisdicción las escuelas del CNE ubicadas, en forma irregular, en las ciudades (MEC, 31-10-1932, p. 156).

Conservadores en sus principios ideológicos, los conductores del CNE entendían que la escuela primaria debía tener una evolución lenta a través del cambio en la organización escolar -pedagógica y didáctica- dejando el modelo enciclopédico para transformarse en un "... verdadero taller de trabajo intelectual y manual, de ambiente amable." La continuidad del cuerpo de inspectores del CNE era un factor fundamental en la permanencia de criterios pedagógicos discretamente paidocéntricos, que defendían una educación “... sin fatiga ni violencia, con la proscripción del sistema represivo, ante la bondad de la disciplina y preventiva del trabajo, suscitada por el interés, la amenidad y por el aspecto utilitario..." nivelando las diferentes de capacidades de aprendizaje (CNE, 1932, p. 47-48).

Las actividades escolares en la Capital Federal seguían influidas por el movimiento renovador de escuela activa y escuela nueva difundido por los inspectores en las dos décadas previas (Puiggrós, 1992; Gvirtz; 1996; Carli, 2006). En esa ciudad, el Instituto "Félix Bernasconi", con sus cuatro escuelas dependientes, fue creado cuatro años antes para ser un centro modelo de experimentación pedagógica, pero la crisis financiera del Estado redujo las posibilidades de emprender las innovaciones previstas -jardín de infantes, sección de retardados, biblioteca infantil- y también se limitaron las experiencias renovación más radicales. No obstante, se ensayaron procedimientos de escuela activa, aplicándose el Plan Dalton, y se inició la creación del Museo Argentino para la Escuela Primaria (CNE, 1932, pp. 100-101).

La reducción global del financiamiento hizo más visible algunas falencias materiales de las escuelas primarias nacionales en todo el país: de los 2.601 locales escolares, la tercera parte era de condición regular y la décima parte mala. Ante la crisis, el CNE inició algunas de las orientaciones sociales de la escuela que continuarían en toda la década: entendían que la escuela fiscal popular necesitaba el apoyo de la sociedad para vencer el analfabetismo, problema cuya falta de resolución provocaba duras 
críticas de los partidos políticos de la oposición, dado que 700.000 niños, el 32\% del total en edad escolar, no recibía educación (La Vanguardia, 2-1-1933, p. 1; 19-11933, p. 1). Las asociaciones cooperadoras, integradas por padres, se encargaron de la asistencia a los niños pobres, distribuyendo guardapolvos y materiales de costura para las labores de las alumnas en las escuelas suburbanas de la ciudad de Buenos Aires. Las nociones de economía doméstica, con contenidos sobre administración del hogar, higiene alimenticia y medicina casera, cobraron mayor utilidad en ese contexto. El papel de las cooperadoras fue valorado, no obstante fueron suprimidas las partidas del presupuesto que tenían asignadas, lo cual redujo su acción a una asistencia alimenticia básica, consistente en la "copa de leche" y un panecillo llamado "la miga de pan" (CNE, 1932, p. 134).

Cumpliendo con la Ley No 1420, que establecía la responsabilidad del Estado en el desarrollo intelectual, moral y físico de los alumnos, la Inspección Médica Escolar consiguió que se crearan veinte "cantinas escolares" -comedores- en la ciudad de Buenos Aires, desde 1928. En agosto de 1932, la Ley N 11.597 las dotó de presupuesto y el CNE proyectó instalarlas también en las provincias y en los territorios nacionales (MEC, agosto 1932, pp. 117-118). Por otro lado, a las "escuelas al aire libre" de esa ciudad asistían 2.500 niños anémicos, la mitad de ellos infectados con tuberculosis. Esta inspección se encargaba de la educación sanitaria mediante conferencias radiales dictadas por médicos, distribución de cartillas y continuas clases informativas efectuadas por 68 visitadoras, que además colaboraron en campañas de vacunación y trabajaron en los comedores escolares (CNE, 1932, pp. 134, 135 y 145).

El CNE debió corregir la herencia del gobierno anterior, que buscó depurar la planta docente y reducir gastos dejando cesante a numerosos maestros y directores de las escuelas primarias nacionales rurales ubicadas en las provincias. Las clases de 1932 se iniciaron con paliativos tales como el trabajo en horarios alternos, refundición de grados paralelos y traslados de personal docente hasta que, en agosto, el CNE consiguió la designación de 118 directores y de 1.529 maestros suplentes, contratados hasta noviembre de ese año. Con los docentes de las escuelas de adultos ocurrió lo mismo pues, de sus 1.893 profesores, 285 quedaron sin empleo durante seis meses. Los pedidos de ascenso del personal también estaban paralizados, habiendo 682 pendientes, de los cuales serían otorgados 521. Estas reparaciones fueron acompañadas de una menor discrecionalidad en las investigaciones sumarias, no obstante, debido a un intento revolucionario de los seguidores del gobierno depuesto, fue decretado el estado de sitio, aumentando la vigilancia estatal en general. Esto afectó el resurgimiento de la actividad gremial de los docentes, como se percibe en las acusaciones del gobernador del Territorio de La Pampa a los maestros de la Asociación de Maestros de la Pampa, tratándolos de anarquistas, inmorales y disolventes -descalificaciones que fueron negadas por éstos-. Los controles oficiales dificultaron también la acción 
sindical docente, liderada por el Frente Único del Magisterio, que buscaba la normalización del pago de salarios organizando un paro (Tierra Libre, 1-5-1932, p. 6-7).

\section{Una renovación paulatina y ordenada de la educación}

El 19 de noviembre fue aceptada la renuncia de los miembros del CNE, y cuatro días después se designó presidente al ingeniero Octavio Pico, político conservador, católico militante y ex ministro del Interior durante el gobierno de facto anterior, ${ }^{2}$ que inició su gestión con fuerte acento personalista, al no estar integrado el cuerpo colegiado que presidía (La Vanguardia, 12-1-1933, p. 1). En los dos meses finales de ese año, tomó sus primeras medidas: encomendó a los inspectores vigilar el cumplimiento de la obligatoriedad escolar, fomentar la creación de cooperadoras, bibliotecas infantiles y populares y organizar actos de carácter moral y patriótico.

El particular nacionalismo que incentivó el CNE tenía adversarios y detractores, especialmente anarquistas, comunistas y socialistas antifascistas. Con el pretexto de un episodio interno de la Asociación del Magisterio de la provincia de Buenos Aires en torno a la infiltración política en la escuela y en un contexto de elecciones de gobernador (La Vanguardia, 1-1-1933, p. 1; 10-3-1033, p. 1.), el 5 de enero de 1933 el presidente del CNE envió una circular en cual prohibía en el ámbito escolar toda manifestación de ideas comunistas, por ser subversiva y contraria a la misión del maestro (MEC, 31 1-1933). Un mes después, recordó al personal docente que les estaba vedada la afiliación, el activismo político y la aceptación de candidaturas para desempeñar cargos electivos, siendo estos motivos de exoneración. Sus derechos políticos se restringían al de ser elector (MEC, 28-2-1933). Los inspectores generales de territorios nacionales y de provincias difundieron la circular, agregando que el control a la propaganda comunista era severo, habiéndose “... extirpado con medidas rápidas y radicales las situaciones anárquicas dentro del personal y todo síntoma de propaganda que atentara contra la estabilidad de nuestras instituciones" (CNE, 1933, p. 425). El órgano de prensa del Partido Socialista, La Vanguardia, ridiculizó la irreal amenaza comunista, por alarmista y exagerada, ya que no tenía anclaje entre los maestros, y acusó la manipulación del nacionalismo como parte de una campaña clerical y xenófoba para desprestigiar a la escuela laica y popular, y para suspender a determinados maestros (La Vanguardia, 9-1-1933, p. 1; 29-1-1933, p. 1). A la vez cuestionó las virtudes cívicas del presidente del CNE, que había sido ministro de la dictadura iniciada con la Revolución de Septiembre, a la cual le seguía rindiendo honores (La Vanguardia, 7-1-1933, p. 1).

Fuera de la censura ideológica, el CNE fue resolviendo algunos problemas burocráticos que afectaban al personal sin alterar el presupuesto: agilizó el pago de los sueldos; ordenó ascensos automáticos de cargo; dio prioridad a la reincorporación de los maestros interinos cesantes por falta de presupuesto; prometió futuros ascensos 
en los territorios nacionales que podían beneficiar hasta el $40 \%$ del personal -1.566 cargos-; reguló la práctica de los embargos de los sueldos, porque eran 1.800 los maestros y empleados del CNE endeudados (MEC, agosto 1933, p. 105; CNE, 1933, pp. 51 y 433). Como contrapartida, los maestros nacionales de varias provincias sufrieron atrasos en el pago de sus salarios de más de seis meses (La Vanguardia, 233-1933, p. 1).

La reforma moderada y progresiva de la organización del trabajo escolar fue iniciada por Octavio Pico y el inspector técnico general, F. J. Picarel en marzo de 1933, en la Capital Federal, cuando aún no estaban designados los demás miembros del CNE, no obstante continuó después de esto. Se proyectó una actualización paulatina de los contenidos de los programas analíticos, respetando el Plan de Estudios -vigente desde 1910-, suprimiendo la sobrecarga de contenidos y aumentando su carácter práctico. Las bases conceptuales oficiales eran cuanto menos eclécticas, porque se enfatizó el papel del conocimiento psicológico como “.... llave para estudiar en el alma del niño el proceso de su despertar a la vida superior, a fin de que el maestro pueda arbitrar los medios para llegar a crear disposiciones que concreten luego en hábitos permanentes."(CNE, 1933, p. 87) La reforma implicó la aplicación de algunas estrategias institucionales y pedagógicas, teniendo como base el concepto de globalización, tratando en forma intensiva un asunto dominante, teniendo en cuenta el orden, las relaciones y dependencias recíprocas de los temas que lo compondrían, sin detenerse en el detalle de los "tópicos". La coordinación de materias afines en torno a un asunto y la correlación entre los grados sucesivos eran otros elementos fundamentales.

Se aconseja vincular las materias afines de cada día y concentrar los distintos aspectos de un asunto por el múltiple interés que suscite su conocimiento y provecho en la observación, la interpretación, la asociación, la aplicación y la construcción, desde los puntos de vista histórico, geográfico, científico y económico y vinculados en lo posible a la producción, a la industria, al comercio y al transporte. (MEC, 31-3-1933, pp. 157-158)

El cambio en la enseñanza requirió la renovación de la tecnología educativa, instalándose dos dispositivos principales: el cuaderno de asuntos y el cuaderno de clase o cuaderno único (Gvirtz, 1999). Cada maestro debía llevar su cuaderno de asuntos, que reemplazaba al cuaderno de tópicos, para planificar el desarrollo de las clases, debiendo constar: a) el enunciado global del asunto central de la clase, respetando los programas; b) los propósitos del maestro y la finalidad práctica de la clase; c) los aspectos a ser asociados en materias; d) la intensidad, extensión y tiempo de los asuntos; e) los recursos técnicos; f) las ilustraciones; g) los ejercicios que el alumno debía hacer en su cuaderno; h) los resultados de las clases anteriores, cotejados con lo 
planificado (CNE, 1933, pp. 94-95). En cuanto a los materiales didácticos, se dispuso que era preferible el estudio a través elementos verdaderos en lugar de los artificiales y que los museos escolares debían convertirse en dinámicos. Los horarios de las clases podrían ser adaptados según las necesidades psico-fisiológicas del alumno, o las actividades del día, pudiéndose también alternar las materias teóricas con las especiales -Labores, Música y Gimnasia-.

En todas las escuelas los inspectores organizaron conferencias sobre la "nueva orientación" de la enseñanza, en las que se trataron todos los instrumentos pedagógicos y administrativos de la reorganización escolar en marcha. (CNE, 1933, p. 145). El CNE aclaraba que con estos cambios organizativos del trabajo escolar había pretendido corregir la improvisación sin alterar los métodos de enseñanza ya consolidados. Si bien el cuaderno único de trabajos diarios del alumno podía permitir la expresión espontánea de sus "potencias espirituales", (CNE, 1933, p. 93) a la vez era un instrumento de control intensivo de esa maduración personal, y un registro "radiográfico" íntimo de su rendimiento, hábitos de orden, estética y capacidad de autocorrección. A la vez facilitaría a la inspección del cumplimiento del programa y de la planificación hecha por el docente.

La coordinación de asuntos y correlación de materias fue aconsejada por la Inspección Técnica General de la Capital Federal, pero su aplicación no fue obligatoria en 1933. Las autoridades del Instituto "Félix Bernasconi" informaron que la integración de materias ya era una práctica en sus escuelas, donde no había ni "régimen carcelario" ni "estática libresca", no obstante fue favorecida por la flexibilidad horaria. Este Instituto implementó el cuaderno de asuntos dando al director de cada escuela la autoridad de unificar los proyectos que presentaran los maestros de cada grado, pero ese año no fue adoptado el cuaderno único. (CNE, 1933, pp. 154-155)

La reforma no implicaba la autonomía del docente o del director para modificar los métodos de enseñanza sin autorización superior, porque debía ser preservada la uniformidad de la enseñanza y su carácter nacionalista. En cuanto a los alumnos, es evidente que el objetivo central de esa educación primaria más eficiente era reforzar el orden social porque, en las mencionadas instrucciones de marzo de 1933, se decía que la escuela primaria debía formar niños buenos, educando más que instruyendo, formando hábitos de trabajo, de sobriedad y de concordia más que inteligencias. (CNE, 1933, p. 91).

\section{La nueva orientación: espiritual, nacionalista y utilitaria}

Los demás miembros directivos del CNE fueron designados el 5 de mayo de 1933: el doctor Félix Garzón Maceda, vicepresidente; los doctores Nicolás Avellaneda y José A. Quirno Costa, y el profesor José Rezzano, vocales. Todos tenían una trayectoria 
pública importante en la política e incluso en la política educativa, heterogénea en términos ideológicos, aunque prevalecía el vínculo con la Iglesia Católica.

El primer gran problema que enfrentaron las autoridades del CNE fue el de la evasión escolar, por el desinterés de los padres, las migraciones laborales y el trabajo infantil. De los 39.005 alumnos primarios de la ciudad de Buenos Aires, ese año sólo el $7 \%$ terminó el $6^{\circ}$ grado, mientras que los demás desertaron principalmente en el primer grado, y progresivamente en los siguientes: $1^{\circ}, 41,5 \% ; 2^{\circ}, 17 \% ; 3^{\circ}, 13,7 \% ; 4^{\circ}$ $11,3 \%$; y 5, 9,5\% (CNE, 1932, p. 120). En la Capital Federal, fue creado un cuerpo de inspectores de obligación escolar, integrado por ochenta maestros encargados de persuadir a los padres y tutores. A los alumnos que no asistían por problemas de alimentación los inscribió en los comedores escolares, y se proporcionó ropa y calzado a quienes no los que no tuvieran. La alimentación dada en las escuelas, aportada por las cooperadoras y por otras instituciones donantes, tuvo un efecto positivo en el aumento de la concurrencia y retención escolar (CNE, 1933, p. 156).

La nueva comisión del CNE se planteó el objetivo de modernizar el funcionamiento administrativo, pero la falta de recursos se lo impidió. Las economías fueron en aumento: se solicitaron rebajas al renovar los contratos de alquiler de locales escolares y no se hicieron nuevos edificios ni refacciones. En los territorios nacionales y en las provincias fueron insuficientes los materiales didácticos, e incluso carecieron de muebles las escuelas recién creadas en locales cedidos o donados. Aún sin recursos, la apertura de escuelas fue intensa: 31 en la Capital Federal, 50 en territorios nacionales y 263 escuelas rurales en las provincias. También fueron importantes los cambios realizados en la reglamentación de la Inspección Médica Escolar, que comprendía colonias de vacaciones, clínica de nutrición, consultorios de especialidades y odontológicos, y sección de educación sanitaria, y se crearon más comedores escolares en la Capital Federal, supervisados por visitadoras de higiene escolar de distrito (CNE, 1933, p. 228), en los cuales se hizo un seguimiento de los alumnos a través de fichas antropométricas -y sociales-. En los comedores se difundieron filmes sobre higiene y las visitadoras debían hacer visitas domiciliarias para comprobar la existencia de enfermedades infectocontagiosas.

Los preceptos pedagógicos que transmitió el CNE mostraban una tensión entre lo tradicional y lo renovador, cuya contradicción epistemológica fue resuelta subordinando los cambios pedagógicos a la cosmovisión espiritualista, nacional y utilitaria, y a ciertas rutinas escolares. Hubo una convivencia de tradiciones de pensamiento pedagógico diferentes, articulándose la espontaneidad con la adaptación al medio y con la moral. La noción de educación integral emergía como superación de la "instrucción", desde una concepción renovada, que incluso incluía elementos discursivos del pensamiento libertario y propuestas del escolanovismo: 
Frente a la escuela estática, libresca, enciclopédica, de tópicos dislocados, en la que el niño se ve condenado a la servidumbre del papel impreso, del debe sin haber, de los trabajos forzados, del régimen penitenciario, del temor inhibitorio que eclipsa la personalidad en el aula fría como celda carcelaria, debe levantarse la escuela dinámica, la escuela funcional, en ambiente de franca espontaneidad... (CNE, 1933, p. 88)

Conseguir que la escuela resultara atractiva al alumno era un objetivo importante también para aumentar la retención escolar. La imagen de una escuela alegre con profesores tolerantes no debía ser contradictoria con una educación nacionalista asociada a hábitos de trabajo, civismo, honradez, disciplina y libertad responsable.

La mejor disciplina es la del trabajo, que suscita la curiosidad y el interés, y que conquista la voluntad y la atención del niño que aprende a dominar sus impulsos instintivos de rebeldía y desorden (...) Educar es adaptar, es formar un estado espiritual, es iluminar la inteligencia, ennoblecer el corazón... (CNE, 1933, p. 87)

El espiritualismo, el espontaneismo, el paidocentrismo, y la preparación para la vida se afincaron en el discurso institucional, consolidándose en los años siguientes. La orientación de la enseñanza primaria puso énfasis en el intento de combinar los principios de la escuela activa inspirada en las ideas de John Dewey con las teorías de las aptitudes para la subsistencia de Herbert Spencer, sin transformar la escuela común en una escuela para el trabajo.

La escuela debía convertirse en intérprete del ambiente del aula, del hogar y de la vida. Bajo la dirección del maestro, el niño debía tener el "maximum de participación activa" para que expresara su personalidad y adquiriera el "sentido de la responsabilidad", es decir el autocontrol (CNE, 1933, p. 88). Se buscaba concretar de este modo una efectiva educación integral "... que armoniza el equilibro físico, intelectual y moral, en la exaltación de los nobles sentimientos, en la ejercitación gradual y razonada de la inteligencia, y en la aplicación constructiva de los recursos prácticos", según palabras de F. J. Picarel (CNE, 1933, p. 93).

La transmisión de conocimientos útiles para la vida se dio de un modo más tangencial. En los territorios nacionales regían los programas de 1911, que buscaban una inclusión de las destrezas laborales en la enseñanza y en algunas escuelas rurales se enseñaban trabajos de huertas. Se sucedieron varias las iniciativas del CNE para dar a las escuelas del campo un currículo práctico con conocimientos agropecuarios, procurando la colaboración del Ministerio de Agricultura de la Nación, y se resolvió destinar 740 pesos mensuales para que los agrónomos regionales, en acuerdo con los directores de las escuelas, compraran herramientas, semillas, árboles y plantas (CNE, 1933, 
pp. 388-389 y 440). Entre las propuestas del CNE sobre unificación de la enseñanza con las provincias se incluyó la enseñanza adaptada a las necesidades económicas y culturales de cada zona, con estímulo de las industrias domésticas e intensificación de la cultura nacionalista (CNE, 1933, pp. 430-431). En las escuelas de adultos también se alentó el aprendizaje de destrezas profesionales y el contacto con la sociedad. La Inspección General de Escuelas de Adultos organizó la Comisión de Ayuda Social Pro Escuelas de Provincias y Territorios para contribuir con útiles, calzado y ropa (CNE, 1933, p. 165) confeccionada por los alumnos de la ciudad de Buenos Aires. También hicieron cien banderas para las escuelas primarias rurales, donadas en un acto público al que asistieron 5.000 alumnos, ante la presencia del Ministro de Guerra, y con el despliegue de dos bandas de música y las compañías de boy scouts de esa capital (MEC, noviembre-diciembre 1933, p. 111).

En 1933, el CNE intensificó el sentimiento nacionalista, a través de la veneración de los símbolos nacionales y el homenaje a los prohombres de la independencia y de la construcción de la Nación en toda ocasión que fuera posible. En un solo año hubo más de treinta conmemoraciones obligatorias en las escuelas del CNE y muchos otros actos específicos de determinadas escuelas, casi en su totalidad con trasfondo patriótico. Las formalidades de los actos y el uso de los símbolos fueron estrictamente pautados y se dispuso que, cuanto menos una vez al mes, los escolares visitaran lugares y monumentos históricos. Las arengas moralistas estaban presentes en todos estos eventos, ya que la educación moral era un propósito establecido por la Ley № 1420. Las celebraciones realizadas en la Capital Federal contaron con la disertación de los más altos funcionarios del organismo, siendo frecuentes los discursos apologéticos apasionados y sobrecargados de alusiones sobre emociones militaristas y conductas virtuosas de los prohombres de la independencia (Escudé, 1990, pp. 107-109).

\section{Conclusiones}

La acción del CNE en el período analizado tuvo continuidad, a través de sus funcionarios de segunda línea, inspectores y directores de reparticiones, con la renovación educacional que se venía desarrollando en las dos décadas anteriores, pero al mismo tiempo la tuvo con la orientación espiritualista y nacionalista del gobierno de facto previo, a través de los integrantes de su órgano de gobierno colegiado. En su combinación ecléctica, esta última vertiente predominó sobre la primera, no obstante los elementos modernizadores de la enseñanza se mantuvieron vigentes así como el discurso liberal progresista acerca de la lucha contra el analfabetismo, en un marco de supervivencia de la principalidad del Estado como educador. La implementación de una nueva organización de la enseñanza tuvo un carácter híbrido, renovadora en lo instrumental pero conservadora en sus objetivos de reproducción del orden social. La crisis económica 
y fiscal limitó la concreción de los proyectos más costosos, dificultó el desarrollo de la infraestructura educativa y afectó las remuneraciones de los educadores, pero a la vez obligó a una mayor conexión de la escuela con la sociedad, al aumentar su función de asistencia material a los alumnos y de acercamiento a sus hogares, sin desviarse del carácter de la educación común e integral fijado por la legislación. El temor latente a un resurgimiento de las ideologías colectivistas opuestas a las instituciones argentinas, esta vez de tendencia comunista, exacerbó el nacionalismo de la conducción educativa, y produjo la reacción de la oposición política, generando un antagonismo que se mantuvo en un plano estrictamente discursivo.

\section{Notas:}

${ }^{1}$ Fue ministro de gobierno de la provincia de Córdoba y diputado nacional por el Partido Autonomista Nacional, director general de Correos y Telégrafos, primer presidente de la Sociedad Rural de Córdoba y promotor del Partido Demócrata de esa provincia, y decano de la Facultad de Agronomía y Veterinaria de la Universidad de Buenos Aires.

${ }^{2}$ Antes fue director de la sección de Tierras y Colonias del Ministerio de Agricultura de la Nación y promotor de los Cursos de Cultura Católica.

\section{Fuentes y Bibliografía}

\section{Fuentes:}

Consejo Nacional de Educación (CNE) (1934). Educación Común en la Capital, Provincias y Territorios Nacionales, Informe presentado al Ministerio de Instrucción Pública por el Consejo Nacional de Educación, año1932, Buenos Aires.

Consejo Nacional de Educación (1935). Educación Común en la Capital, Provincias y Territorios Nacionales, Informe presentado al Ministerio de Instrucción Pública por el Consejo Nacional de Educación, año1933, Buenos Aires.

El Monitor de la Educación Común (MEC) (1932-1933). Revista mensual, órgano del Consejo Nacional de Educación, Buenos Aires.

La Vanguardia (1933). Diario oficial del Partido Socialista de la Argentina, Buenos Aires.

Tierra Libre (1932). Periódico anarquista, Bahía Blanca.

\section{Referencias}

Ascolani, A. (2015). Ruralidad, analfabetismo y trabajo en la Argentina. Proyectos y acciones del Consejo Nacional de Educación (1930-1940). Cadernos de História da Educação, 3 (14), 853-877.

Ascolani, A. (2017). Concepciones reformistas en torno a las funciones de la Educación Primaria Rural Argentina (1930-1960). Dialogia 2, 43-68. 
Ascolani, A. \& Gindin, J. (2018). Sindicalismo docente en la Argentina y el Brasil. Procesos históricos del siglo XX. Rosario: Laborde Editor.

Bejar, M, D. (1992). Altares y banderas en una educación popular: la propuesta del gobierno de Manuel Fresco en la Provincia de Buenos Aires (1936-1940). Estudios e Investigaciones, 12, 83-130.

Carli, S. (2006). Niñez, Pedagogia y Política. Buenos Aires: Miño y Dávila.

Escudé, C. (1990). El fracaso del proyecto argentino. Educación e ideología. Buenos Aires: Editorial Tesis.

Gvirtz, S. (Comp.) (1996). Escuela Nueva en Argentina y Brasil. Visiones comparadas. Buenos Aires: Miño y Dávila.

Gvirtz, S. (Comp.) (1999). El discurso escolar a través de los cuadernos de clase. Argentina (1930-1970). Buenos Aires: EUDEBA.

Ossanna, E. (Coord.) (1993). La educación en las provincias (1985-1945). Buenos Aires: Galerna.

Puiggrós, A. (1992). La Educación argentina desde la Reforma Saavedra Lamas hasta el fin de la década infame. In: A. Puiggrós, A. (Dir.). Escuela, Democracia y Orden (1916-1943). (pp. 121-134). Buenos Aires: Galerna.

\section{Adrián Ascolani}

Doctor en Historia por la Universidad Nacional de La Plata. Director del Instituto Rosario de Investigaciones en Ciencias de la Educación, Consejo Nacional de Investigaciones Científicas y Técnicas. Profesor Titular de la Universidad Nacional de Rosario, Argentina. Email: aascolani@yahoo.es

Correspondência Instituto Rosario de Investigaciones en Ciencias de la Educación Adrián Ascolani Ocampo y Esmeralda - Predio CONICET Rosario Santa Fe - Argentina

Data de submissão: Março 2018 Data de avaliação: Agosto 2018 Data de publicação: Abril 2019 